\title{
Differential distributions for top-quark pair production at NNLO
}

\author{
David Heymes* \\ Cavendish Laboratory, \\ University of Cambridge, \\ Cambridge CB3 OHE, UK \\ E-mail: dheymes@hep.phy.cam.ac.uk
}

Differential distributions for top-quark pair production at the LHC at next-to-next-to-leading order in perturbative QCD are presented. Appropriate dynamical scales for the factorization and renormalization scales are identified, which ensure perturbative convergence in a wide kinematical regime. A selection of precision results for the main differential distributions, that are measured at the LHC, are shown.

Loops and Legs in Quantum Field Theory

24-29 April 2016

Leipzig, Germany

${ }^{*}$ Speaker. 


\section{Introduction}

Top quarks are predominantly produced in pairs in hadron collisions. Therefore, top-quark pair production is an important process to study top-quark properties at the LHC. Inclusive measurements at 7, 8 and $13 \mathrm{TeV}$ have been published in $[1,2,3,4,5,6]$. Differential results have been presented in $[7,8,9]$. The experimental precision some of these measurements has reached below $10 \%$ level and is expected to improve further in the near future. Therefore, precision predictions for top-quark pair production are indispensable in order to test the Standard Model to the experimental accuracy that is reached at the LHC. Small deviations from the Standard Model are only noticeable if predictions within the Standard Model are robust and precise.

The total inclusive cross section has been predicted at next-to-next-to-leading order (NNLO) including soft gluon effects to all orders at next-to-next-to-leading log (NNLL) accuracy [10] and shows good agreement with measurements at 7,8 and $13 \mathrm{TeV}$. At the differential level several approximate NNLO predictions have been presented. They include the full next-to-leading order (NLO) result and partial contributions of the NNLO [11, 12, 13]. We remark that the approximate results of Ref. [13] stated as approximate NNNLO, do not include the full NNLO result and are formally only an approximation of the NNLO result.

As far as the full NNLO is concerned, the first differential result has been the forward-backward asymmetry at the Tevatron [14]. It has been obtained by the same methods and a modification of the software that has been used previously for the total inclusive cross section. Subsequently, the same implementation could be applied to perform a complete study of kinematic distributions which have been measured at the Tevatron [15]. This first implementation was based on the subtraction scheme STRIPPER $[16,17]$, but turned out to be not powerful and flexible enough to deal with the increasing demands of the LHC. Therefore, a complete new Monte-Carlo event generator has been developed based on the improved and complete general formulation of the subtraction scheme STRIPPER [18]. First differential predictions for the LHC at $8 \mathrm{TeV}$ have been published [19].

All aforementioned results at NNLO have been obtained for a fixed-scale choice $\mu=m_{t}$, where $m_{t}$ is the mass of the top quark. This allowed only to provide reliable predictions in a limited kinematical regime. Recently, extended predictions at NNLO for the LHC at $8 \mathrm{TeV}$ and $13 \mathrm{TeV}$ using dynamical scales have become available [20]. In this write-up, we present the appropriate scale choice for differential distributions for top-quark pair production at fixed order perturbative QCD. This presentation in section 2 is based on the LHC setup at $8 \mathrm{TeV}$. Afterwards, we show selected results for the LHC setup at $13 \mathrm{TeV}$ in section 3.

\section{Scale choice for top-quark pair production at fixed order perturbative QCD}

In fixed-order perturbative QCD the cross-section depends on the factorization scale $\mu_{F}$ and renormalization scale $\mu_{R}$

$$
\sigma_{h_{1} h_{2}}\left(P_{1}, P_{2}\right)=\sum_{a b} \iint_{0}^{1} \mathrm{~d} x_{1} \mathrm{~d} x_{2} f_{a / h_{1}}\left(x_{1}, \mu_{F}^{2}\right) f_{b / h_{2}}\left(x_{2}, \mu_{F}^{2}\right) \hat{\sigma}_{a b}\left(x_{1} P_{1}, x_{2} P_{2} ; \alpha_{s}\left(\mu_{R}^{2}\right), \mu_{R}^{2}, \mu_{F}^{2}\right) .
$$

The residual dependence on these scales is used to estimate the error due to neglecting higher orders in the perturbative expansion of the partonic cross section $\hat{\sigma}_{a b}$ in $\alpha_{s}$. Therefore, it quantifies the 


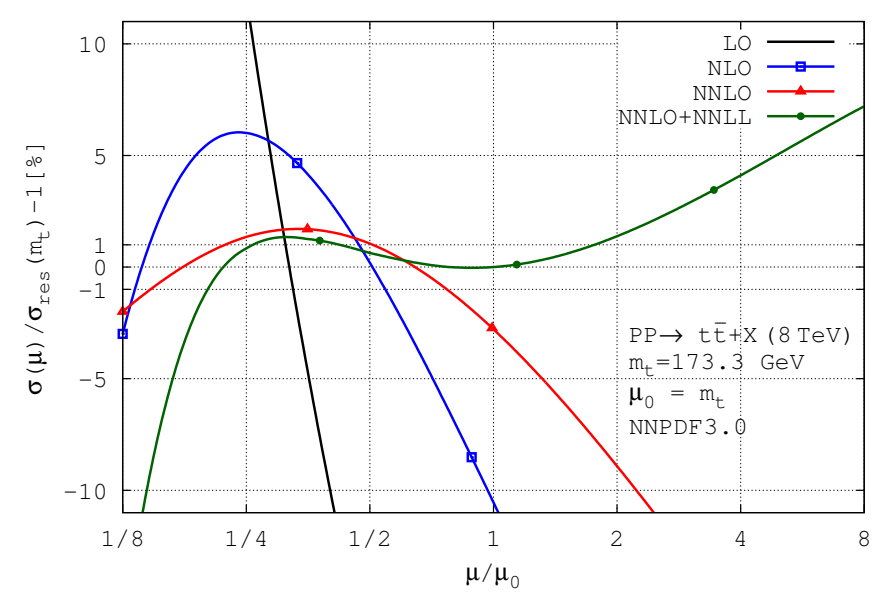

Figure 1: Total inclusive production cross section of a top-quark pair as function of the renormalization and factorization scale $\mu=\mu_{F}=\mu_{R}$ at $8 \mathrm{TeV}$. The predictions at different orders of perturbation theory are normalized to the NNLO+NNLL prediction $\sigma_{\text {res }}(\mu)$ at $\mu=m_{t}$.

theoretical uncertainty of the predictions. This scale uncertainty is obtained by varying the renormalization and factorization scale independently between half and two a central scale $\mu_{0}$. However, the choice of the central scale is ambiguous in contrast to all other input parameters which can be fixed by independent measurements, e.g. $\alpha_{s}$ and $m_{t}$.

All previous calculations for top-quark pairs at NNLO have been performed using a fixed central scale, i.e. $\mu_{0}=m_{t}$. This is a natural choice for the total inclusive cross section, which only depends on the top-quark mass for a fixed collider energy. However, differential distributions can incorporate an additional scale, e.g. the transverse momentum distribution depends on the transverse momentum $p_{\mathrm{T}}$. This additional dependence can be incorporated in an appropriate dynamical scale $\mu\left(p_{\mathrm{T}}\right)$.

We give a short summary of how a good dynamical scale for differential distributions can be picked and restrict ourselves in this write-up to predictions at $8 \mathrm{TeV}$. Results at $13 \mathrm{TeV}$ using these scales are presented in the next section. A more detailed discussion of different dynamical and fixed scales for top-quark pair production at 8 and $13 \mathrm{TeV}$ has been performed in Ref. [20] and high quality predictions are available.

The main guidance for choosing a scale is convergence of the perturbative series of the total inclusive cross section and of differential distributions. First, the total inclusive cross section as a function of the scale is considered, which is shown in Fig. 1. The best available prediction is at NNLO and resums soft-gluon effects to NNLL. It is denoted by $\sigma_{\text {res }}(\mu)$. The point of fastest convergence can be read off the diagram as the value of $\mu$ that satisfies $\sigma_{\mathrm{NNLO}}(\mu)=\sigma_{\text {res }}(\mu)$. This point lies slightly above $\mu=1 / 2 m_{t}$. Soft-gluon resummation has a negligible impact on the fixed order NNLO result at this point. Additionally, the total cross section at this point at NNLO is approximately equal (within $1 \%$ ) to the best prediction of the total inclusive cross section, which is $\sigma_{\text {res }}\left(\mu=m_{t}\right)$.

This point of fastest convergence is now used as a guidance for the limiting behaviour of the dynamical scale in the lower kinematical region of the differential distribution. We discuss the invariant 
mass distribution of the top-quark pair $m_{t \bar{t}}$ and the transverse momentum distribution $p_{T}$ of the top and the antitop. Since the bulk of the total cross section comes from the threshold region of the $m_{t \bar{t}}$-distribution and the low $p_{T}$-region of the $p_{T}$-distribution, a dynamical scale should behave as $\sim 1 / 2 m_{t}$ in these regions. In the tails of the distributions, corresponding to $p_{T} \gg m_{t}$, the behaviour of the scale is commonly chosen to be proportional to the $p_{T}$. Again, perturbative convergence can be used to determine the explicit form. Here, we state the conclusions of the complete discussion [20]. In Fig. 2 the two differential distributions for wide kinematical regimes are presented. The
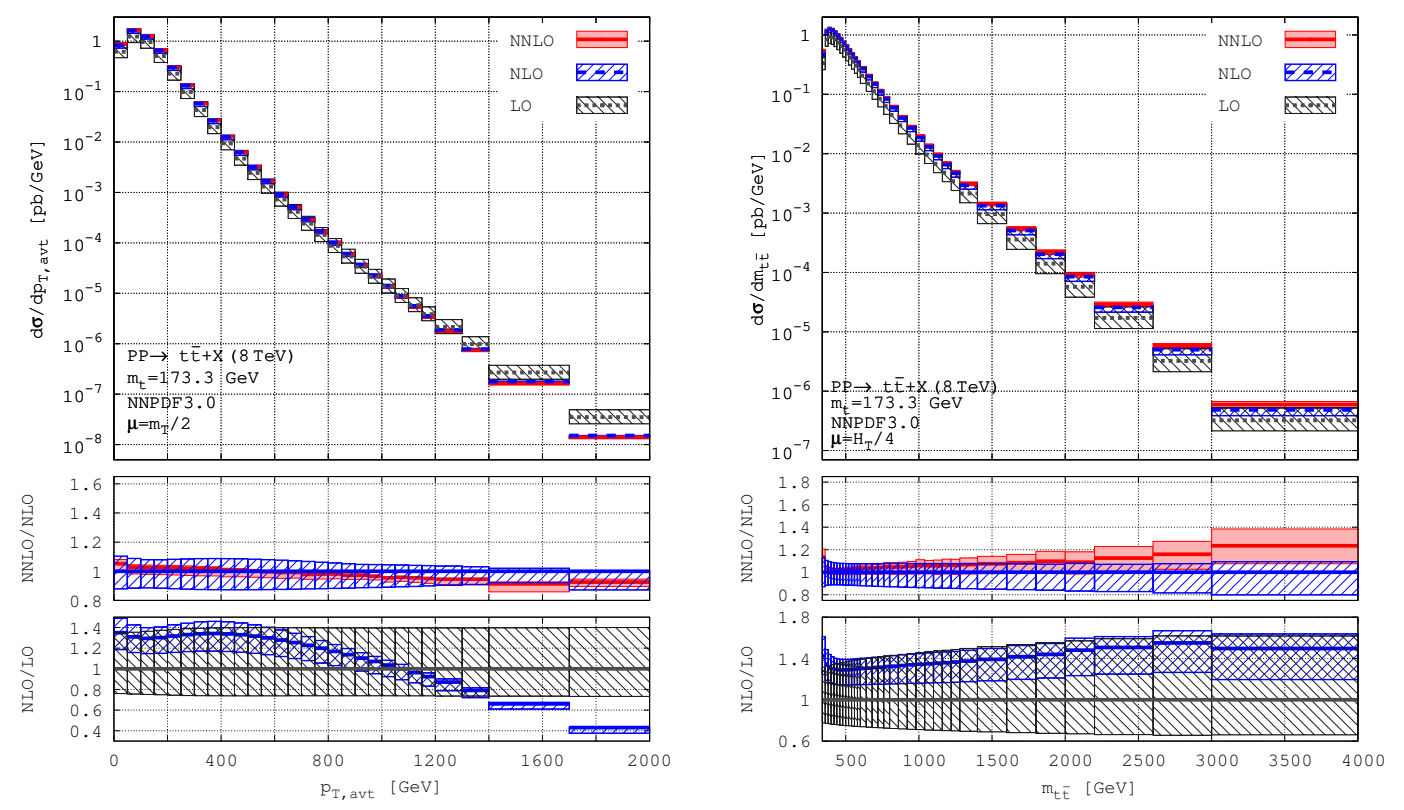

Figure 2: The average $p_{T, t / \bar{t}}$ (left) and $m_{t \bar{t}}$ distributions (right) in leading order (LO), next-to-leading order (NLO) and next-to-next-to-leading order (NNLO) QCD at LHC at $8 \mathrm{TeV}$. Error bands are from scale variation.

appropriate scale for the $p_{\mathrm{T}}$-distribution is

$$
\mu=\mu_{0}=\frac{1}{2} m_{\mathrm{T}}(t / \bar{t}) \quad \text {,with } \quad m_{\mathrm{T}}(t / \bar{t})=\sqrt{m_{t}^{2}+p_{\mathrm{T}, t / \bar{t}}^{2}},
$$

where $m_{\mathrm{T}}(t)$ is chosen for the $p_{\mathrm{T}}$-distribution of the top quark and $m_{\mathrm{T}}(\bar{t})$ is chosen for the $p_{\mathrm{T}}$ distribution of the antitop quark. The left plot of Fig. 2 shows the average of the two distributions. Using this scale, the NNLO prediction lies within the error band of the NLO prediction in the whole $p_{\mathrm{T}}$-range. By looking at the NNLO K-factor we observe that the theoretical uncertainty is significantly reduced. Hence, a precise prediction is obtained by maintaining perturbative convergence. Additionally, we observe by looking at the NLO K-factor for large $p_{\mathrm{T}}$ values, that it is essential to include NNLO prediction to draw conclusions for a specific scale.

For the $m_{t t}$-distribution the appropriate central scale reads

$$
\mu=\mu_{0}=H_{T} / 4 \quad \text {, with } \quad H_{T}=\sqrt{m_{t}^{2}+p_{\mathrm{T}, t}^{2}}+\sqrt{m_{t}^{2}+p_{\mathrm{T}, \bar{t}}^{2}} .
$$

By looking at the NNLO K-factor on the right hand side of Fig. 2, we observe a good perturbative convergence up to large values of $m_{t \bar{t}}$. The scale $\mu=H_{T} / 4$ also preserves perturbative convergence 
of the rapidity distribution of the top quark (antitop quark) and the rapidity distribution of the topquark pair.

The behaviour of these two scales at low $p_{\mathrm{T}}, \mu\left(p_{\mathrm{T}} \rightarrow 0\right) \rightarrow 1 / 2 m_{t}$, ensures that the total inclusive cross sections $\sigma_{\mathrm{NNLO}}\left(H_{T} / 4\right)$ and $\sigma_{\mathrm{NNLO}}\left(m_{\mathrm{T}} / 2\right)$ are numerically equal to $\sigma_{\mathrm{res}}\left(m_{t}\right)^{1}$.

\section{Phenomenology at $13 \mathrm{TeV}$ LHC}
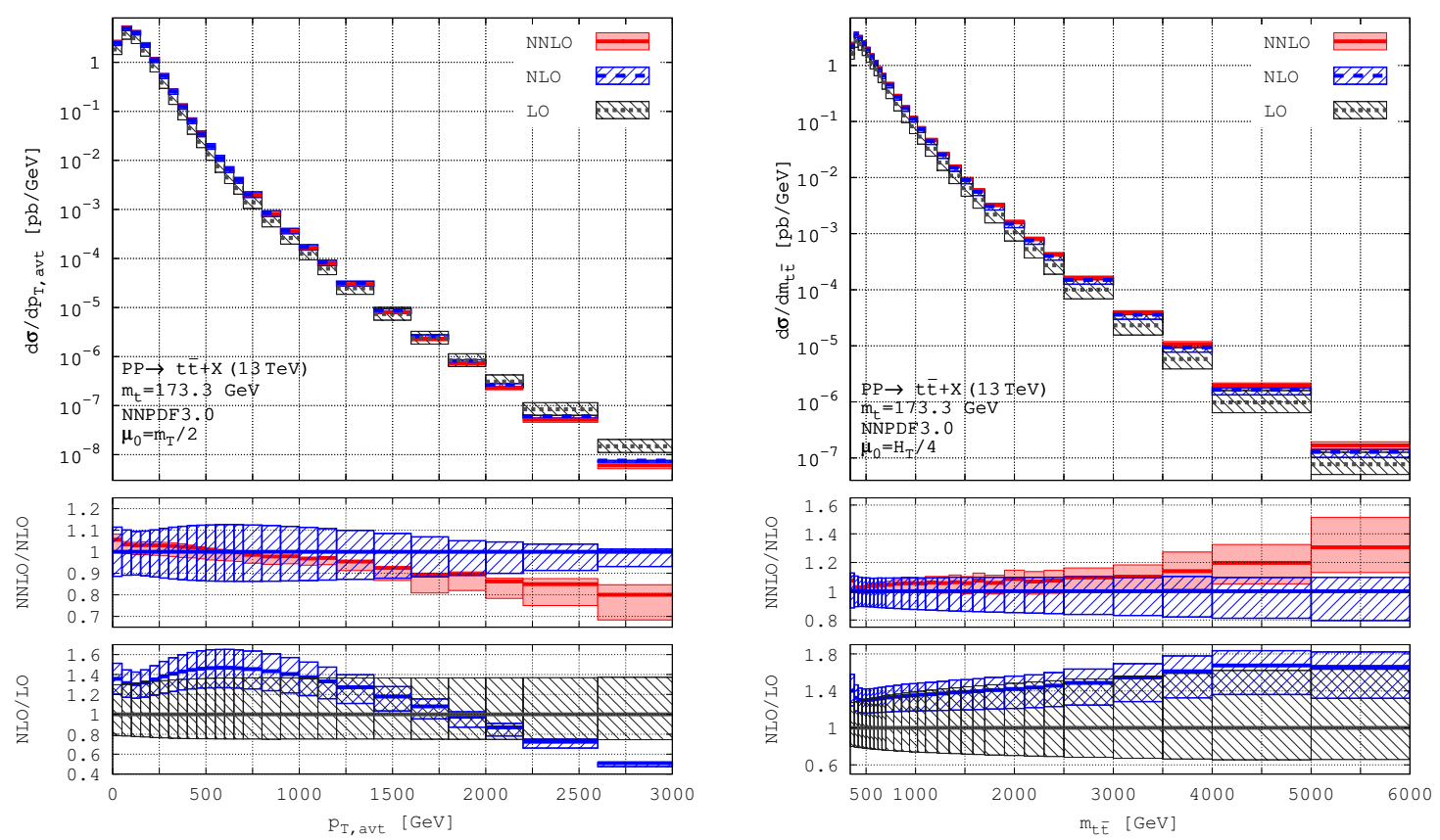

Figure 3: The average $p_{T, t / \bar{t}}$ (left) and $m_{t \bar{t}}$ (right) distributions at LO, NLO and NNLO QCD at the LHC at $13 \mathrm{TeV}$. Error bands are from scale variation.

Once a appropriate central scale has been determined, as explained in the previous section, high precision predictions can be obtained. Here, we present predictions for differential observables for stable top quarks, that have been measured so far at the LHC. While all available predictions can be found in [20], we show selected results for the LHC at $13 \mathrm{TeV}$ in Figs. 2 and 3 using the NNPDF3.0 pdf set [21].

Fig. 3 (left) shows the $p_{T, t / t}$-distribution up to $3 \mathrm{TeV}$. For this distribution the NNLO central value typical lies within the error band of the NLO scale uncertainty. Only at very large values of the transverse momentum, the NNLO central value lies outside the NLO error band. The NLO uncertainty band underestimates the error in this region. Additional, in this region collinear logs of the form $\log \left(p_{\mathrm{T}} / m_{t}\right)$ could become important and should be resummed to all orders.

Fig. 3 (right) shows the $m_{t t}$-distribution in the kinematic regime up to $6 \mathrm{TeV}$. This distribution shows good perturbative convergence in almost the full kinematic regime. Only beyond $3.5 \mathrm{TeV}$ the central value of the NNLO cross section lies outside the error band of the NLO cross section. However, the error NLO and NNLO error bands still overlap, which indicates perturbative convergence in this region as well.

\footnotetext{
${ }^{1}$ The relative difference is below $1 \%$
} 

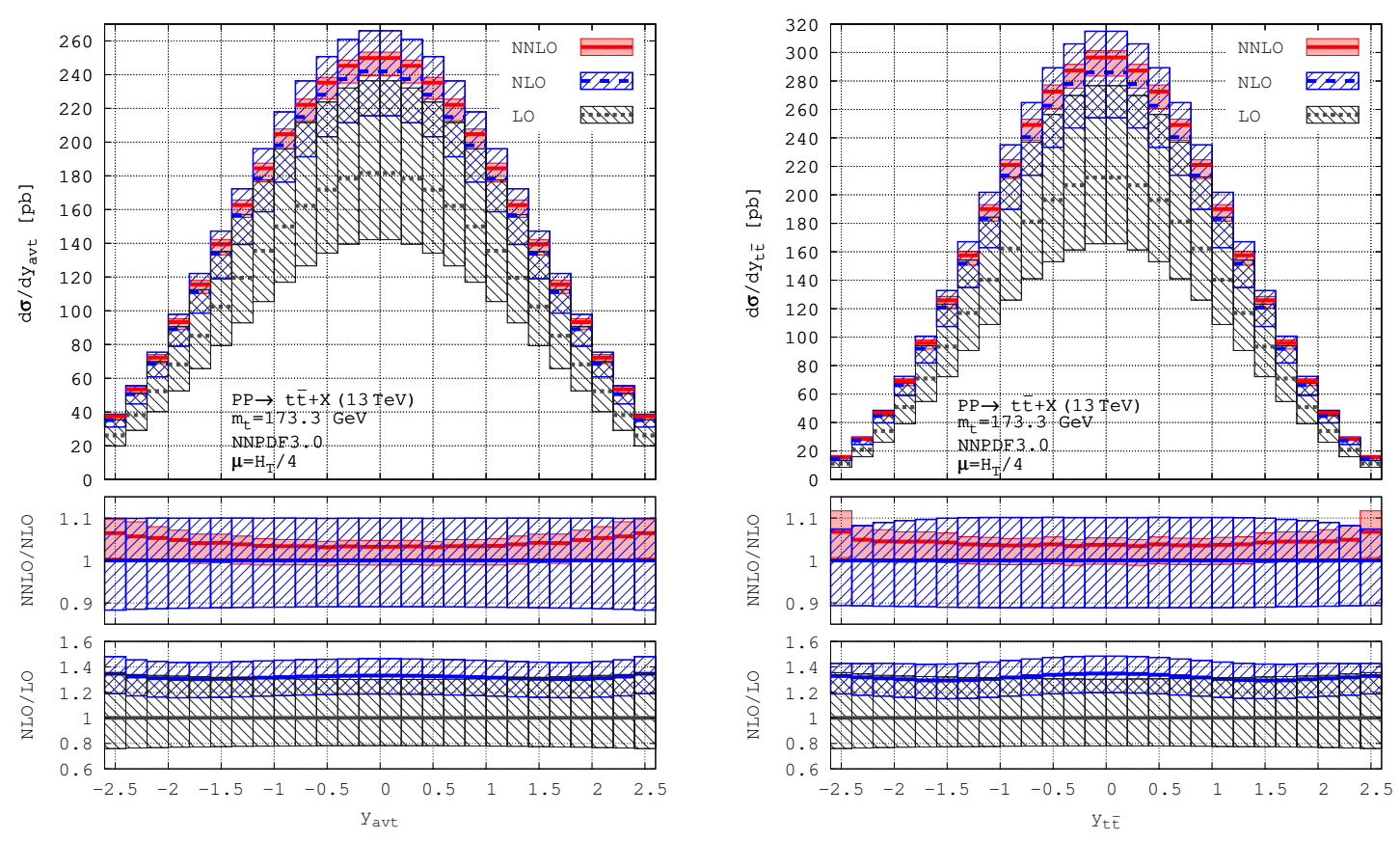

Figure 4: The average $y_{t / \bar{t}}$ (left) and $y_{t \bar{t}}$ (right) distributions at LO, NLO and NNLO QCD at the LHC at $13 \mathrm{TeV}$. Error bands are from scale variation.

For both aforementioned distributions $\left(p_{T, t / \bar{t}}\right.$ and $\left.m_{t \bar{t}}\right)$ the presented predictions are of high quality. The covered kinematical range is beyond what is currently measured at the LHC and will be, most likely, sufficient for the long-term run of the LHC.

In Fig. 4 the $y_{t / \bar{t}}$ and the $y_{t \bar{t}}$ distributions are shown. The NNLO result lies within the scale band of the NLO result for both distributions in the full displayed regime, which again indicates good perturbative convergence. As can be observed in Fig. 4 both results are of high quality.

\section{Conclusion and outlook}

In this write-up we have motivated dynamical scales, that are most appropriate to predict differential distributions for top-quark pair production in fixed order perturbative QCD. The analysis is based on perturbative convergence of the total inclusive as well as the differential cross section. We find that for the $m_{t \bar{t}}$-distribution as well as the rapidity distributions the central scale choice $\mu=H_{T} / 4$ satisfies all requirements. For the $p_{\mathrm{T}}$-distribution the central scale choice $m_{\mathrm{T}} / 2$ turns out to permit reliable predictions up to large values of $p_{\mathrm{T}}$. Using these scales high quality predictions have been presented for the LHC at $13 \mathrm{TeV}$. We have not computed the pdf uncertainty and want to refer to [20] for a further discussion of a anticipated pdf uncertainty, which is expected to be large in the multi-TeV range of differential distributions. These NNLO results are currently the most precise predictions for stable top-quark pairs. The presented scale uncertainty, estimating the error due to neglecting higher orders, is typically at the order of 5\%, but varies in different kinematical regimes and across different distributions.

A next level of improvement can be expected by including electroweak corrections. Their impact 
on the cross section in different kinematical regimes will be investigated in the near future. A crucial next step is the inclusion of top-quark decays at NNLO [22, 23]. This would allow to simulate the experimental setup more realistically.

\section{Acknowledgments}

I thank the organizers for the possibility to present these results at Loops and Legs 2016. I would like to thank my collaborators M. Czakon and A. Mitov. This work is supported by the UK Science and Technology Facilities Council [grant ST/L002760/1].

\section{References}

\section{References}

[1] V. Khachatryan et al. [CMS Collaboration], arXiv:1603.02303 [hep-ex].

[2] G. Aad et al. [ATLAS Collaboration], Eur. Phys. J. C 74, no. 10, 3109 (2014)

[3] V. Khachatryan et al. [CMS Collaboration], [arXiv:1602.09024 [hep-ex]].

[4] G. Aad et al. [ATLAS Collaboration], Phys. Rev. D 91, no. 11, 112013 (2015)

[5] ATLAS collaboration ATLAS-CONF-2016-005

[6] V. Khachatryan et al. [CMS Collaboration], Phys. Rev. Lett. 116, no. 5, 052002 (2016)

[7] G. Aad et al. [ATLAS Collaboration], arXiv:1511.04716 [hep-ex].

[8] V. Khachatryan et al. [CMS Collaboration], Eur. Phys. J. C 75, no. 11, 542 (2015)

[9] TOP-16-008, TOP-16-011

[10] M. Czakon, P. Fiedler and A. Mitov, Phys. Rev. Lett. 110, 252004 (2013)

[11] M. Guzzi, K. Lipka and S. O. Moch, JHEP 1501, 082 (2015)

[12] B. D. Pecjak, D. J. Scott, X. Wang and L. L. Yang, arXiv:1601.07020 [hep-ph].

[13] N. Kidonakis, Phys. Rev. D 91, no. 3, 031501 (2015)

[14] M. Czakon, P. Fiedler and A. Mitov, Phys. Rev. Lett. 115, no. 5, 052001 (2015)

[15] M. Czakon, P. Fiedler, D. Heymes and A. Mitov, JHEP 1605, 034 (2016)

[16] M. Czakon, Nucl. Phys. B 849, 250 (2011)

[17] M. Czakon, Phys. Lett. B 693, 259 (2010)

[18] M. Czakon and D. Heymes, Nucl. Phys. B 890, 152 (2014)

[19] M. Czakon, D. Heymes and A. Mitov, Phys. Rev. Lett. 116 (2016) no.8, 082003

[20] M. Czakon, D. Heymes and A. Mitov, arXiv:1606.03350 [hep-ph].

[21] R. D. Ball et al. [NNPDF Collaboration], JHEP 1504, 040 (2015) doi:10.1007/JHEP04(2015)040 [arXiv:1410.8849 [hep-ph]].

[22] M. Brucherseifer, F. Caola and K. Melnikov, JHEP 1304, 059 (2013)

[23] J. Gao, C. S. Li and H. X. Zhu, Phys. Rev. Lett. 110, no. 4, 042001 (2013) 